Revista Española de Antropología Americana ISSN: 0556-6533

https://doi.org/10.5209/reaa.64965

\title{
De cabezas y lenguas en los reinos mayas. Cambios versus permanencia durante y tras el colapso
}

\author{
Vera Tiesler ${ }^{1}$ y Alfonso Lacadena García-Gallo ${ }^{2}$
}

Recibido: 14 de diciembre de 2019 / Aceptado: 1 de abril de 2019

Resumen. Las modificaciones cefálicas artificiales en infantes estuvieron a cargo de las mujeres y fueron algunas de las prácticas más difundidas entre los mayas prehispánicos, con resultados tan diversos como visibles en los semblantes de los mayas del periodo Clásico. En este artículo se exploran sus significaciones identitarias, potencialmente étnicas, a través de los territorios mayas y más allá. Para este fin hemos revisado la cartografía de los vestigios humanos mayas, sus retratos y las inscripciones, los cuales en parte siguen la distribución de su habla en territorios políticos cambiantes y cada vez más divididos. Nuestras indagaciones trazan similitudes entre lenguas y formas cefálicas que a su vez dejan entrever antagonismos. Las diferencias se vuelven especialmente patentes entre los hablantes del ch'olano occidental, con sus testas alargadas e inclinadas, y aquellos de habla tzeltalana, zoque y k'iche', quienes solían lucir una cabeza corta y ancha.

Palabras clave: modificación cefálica; maya; lenguas vernaculas; etnicidad; colapso.

\section{[en] Of Head Shapes and Languages among the Maya Kingdoms. Shifts versus Permanence Towards and Past the Collapse}

\begin{abstract}
The artificial modification of head form, conferred by females upon their offspring, was a widespread physical enhancement with diverse visible results among the Classic-period Maya. This paper explores the roles of culturally conferred head shapes in the public display of group identity (potentially ethnicity) across and beyond the Maya territories. To this end, we survey the area's head portraiture and cranial shapes together with the inferred vernacular languages. While narrow, inclined, and elongated heads were prominently displayed among the Western Ch'olan around the Usumacinta basin and bordering Tzeltalan, Zoque and K'iche' communities sported broad and shortened heads. In the context of an increasingly divided political landscape surrounding the Maya collapse, we discuss the shifting scope of head shapes in terms of identity vs. alienation, interaction vs. antagonism.
\end{abstract}

Keywords: cranial vault modification; Maya; spoken languages; ethnicity; collapse.

Sumario. 1. Introducción. 2. Las formas cefálicas entre los reinos mayas del Clásico. 3. De siluetas cefálicas mayas y lenguas vernáculas durante el Clásico. 4. Cambios versus permanencia tras el ocaso de las hegemonías mayas. 5. De modificaciones cefálicas y mitos de creación humana. 6. Conclusiones. 7. Referencias.

Cómo citar: Tiesler, Vera y Alfonso Lacadena García-Gallo. 2019. «De cabezas y lenguas en los reinos mayas. Cambios versus permanencia durante y tras el colapso». Revista Española de Antropología Americana 49 (número especial): 157-181.

\footnotetext{
1 Facultad de Ciencias Antropológicas, Universidad Autónoma de Yucatán. vtiesler@yahoo.com

2 Departamento de Historia de América y Medieval y Ciencias Historiográficas. Universidad Complutense de Madrid.
} 


\section{Introducción}

Han pasado al menos dos décadas desde que Alfonso Lacadena y Søren Wichman sospecharon que ya durante el primer milenio d.C. se hablaban más idiomas en las Tierras Bajas mayas que solo aquella lengua franca que servía a los cortesanos para escribir e inscribir. Al analizarse las versiones ortográficas de la escritura clásica maya -sitio por sitio y atravesando los vastos territorios de esta esfera- todo parece indicar que las inflexiones presentaban una diversidad geográfica en el habla. Fue de este modo que Lacadena y Wichman procedieron a delimitar territorios donde la gente debiera haber conversado en yucateco y otros, más al sur, donde se hablaba el ch'olano occidental a lo largo del río Usumacinta y ch'olano oriental hacia el este; para convertirse en quiché y en tzeltalano subiendo las cadenas montañosas (Lacadena y Wichman 2002).

Precisamente esta nueva cartografía lingüística, con sus potenciales significados culturales, fue el punto de partida para un diálogo interdisciplinario entre ambos autores, motivado por la curiosidad por escudriñar si el caleidoscopio vernacular maya del Clásico guardaba coincidencias con las formas cefálicas de sus hablantes y cómo lo hacía (Tiesler y Lacadena 2018). Aunque tal confrontación pudiera antojarse atrevida, no lo es en realidad al tratarse de dos tradiciones culturales vinculadas íntimamente con la infancia, la permanencia cultural y el hogar. Al igual que en otras partes de la gran Mesoamérica, era en la esfera doméstica donde los chicos aprendían a entender y conversar en su lengua materna y donde las madres reproducían las credenciales grupales en la testa de sus pequeños.

Las modificaciones de la bóveda craneal describen una práctica nativa profundamente arraigada que se remonta a miles de años de tradición, parte realmente de lo que Alfredo López Austin (2001) denominó como «núcleo duro» del pensamiento nativo. El registro arqueológico de restos craneales da testimonio de su antigüedad, ubicando los cráneos modificados mayas más tempranos entre los períodos Preclásico Temprano y Medio (Saul 1972). A diferencia de las modas más efímeras expresadas en vestimenta y arreglo corporal, el modelado cefálico, impreso en el menor, resulta ser permanente, condición que le proporciona una calidad más conservadora. Su promulgación (aunque no necesariamente su exhibición) se suscribía al mundo de la reproducción cultural femenina. Esta idea encuentra confirmación al pensar en los muchos retratos prehispánicos y relatos etnohistóricos de las prácticas cefálicas (Tiesler 2014; Zabala 2014; Pérez 2018).

En nuestra presente incursión confrontaremos el caleidoscopio geográfico de las formas craneanas en el registro arqueológico de sitios y regiones maya del Clásico, con las siluetas cefálicas de los retratos, con la distribución inferida de las lenguas habladas y las relaciones políticas entre los reinos que se disputaban el dominio en las Tierras Bajas mayas. La fructífera combinación de estos enfoques está diseñada para poner al descubierto nuevas formas (algunas ocultas) de reproducción cultural y sentido de pertenencia, identidad y, en algunos casos, «etnicidad» entre los mayas del periodo Clásico ${ }^{3}$. Con este modus operandi invitaremos además al debate sobre

\footnotetext{
3 Para nuestros propósitos, el término «etnicidad» denota formas específicas de cohesión grupal y afiliación que acompaña la identificación propia de la población en oposición a «otras». De tal modo, los miembros de un grupo étnico se identifican entre sí, generalmente sobre la base de una genealogía y ascendencia común, que puede ser mítica, presumida o real. Otros vínculos grupales suelen basarse en el idioma, la religión y/o mitos de origen compartidos (Barth 1969; véase también Díaz-Andreu et al. 2005). Quienes conciben una afinidad de este tipo tienden
} 

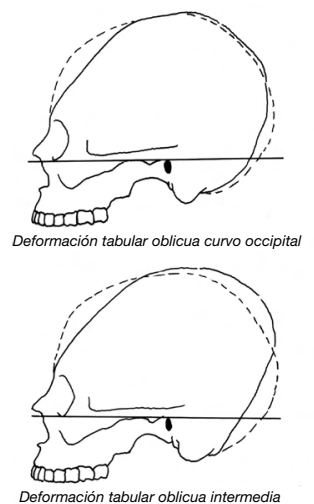

Deformación tabular oblicua intermedia

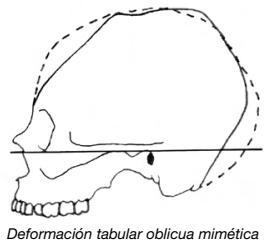

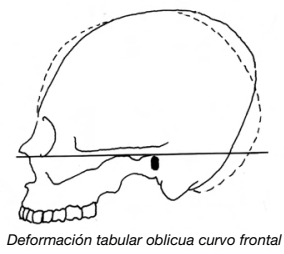

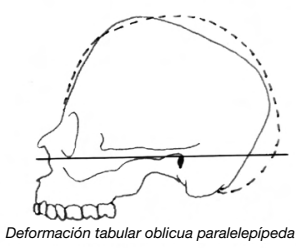

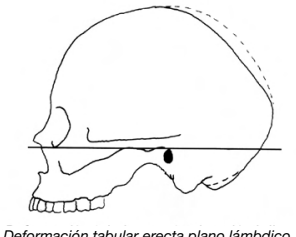

Deformación tabular erecta plano lámbdico

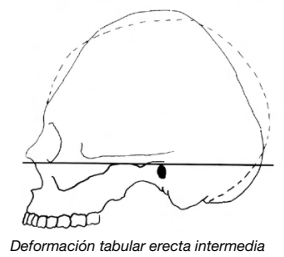

Deformación tabular erecta intermedia

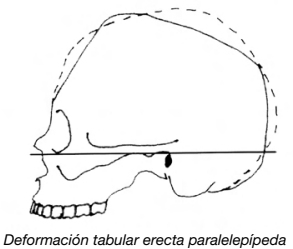

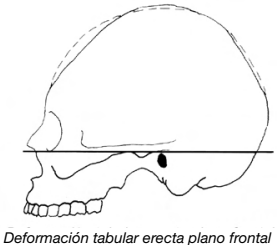

Deformación tabular erecta plano frontal

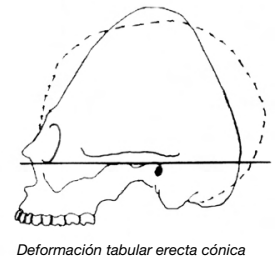

Figura 1. Cuadro taxonómico de Imbelloni (Dembo e Imbelloni 1938: 275), adaptado para diferenciar los tipos básicos y variantes de las modificaciones tabulares oblicuas (izquierda) y erectas (derecha) (morfología esquemática; trazo de Vera Tiesler).

los mecanismos intrínsecos sociales, propios de cambio sociopolítico, de reemplazo versus resiliencia y permanencia, tal como nos lo muestra una era de tránsito profundo en el pasado maya y mesoamericano: el «Epiclásico» o «Clásico Terminal».

\section{Las formas cefálicas entre los reinos mayas del Clásico}

Para examinar las formas cefálicas mayas durante el Clásico recurrimos a una base de datos que contiene información contextualizada de 1.205 individuos con cráneos evaluables, más otros estudios de antropología física que se hayan publicado o reportado por escrito. Estos resultados cubren el sur y sureste mexicano, Guatemala, Belice y Honduras (Wright 1997; Wright y Witte 1998; Scherer et al. 1999; Montes 2000; Scherer y Wright 2001; Scherer 2006, 2007, 2008, 2015, 2018; Núñez 2009, 2010; Tiesler 2012, 2014). En cada serie se examinaron los aspectos de sexo, edad, cronología y estatus social, presencia-ausencia de modelación cultural y, en los individuos modificados, las técnicas e implementos compresores, los grados de modificación y las formas particulares que resultasen (para mayor información sobre los procedimientos de clasificación, véase Tiesler 2012) (Figura 1).

Nótese que, lejos de representar equitativamente los segmentos cronológicos del Clásico, las poblaciones esqueléticas están sesgadas fuertemente hacia el Clásico

a identificarse entre sí y expresar su adhesión a través de prácticas culturales, glosas y creencias compartidas, a veces en confrontación con otros. Si bien sigue estando debatida (Jones 1997), tal demarcación al menos facilita formular preguntas discursivas junto con aquellas asociadas al físico, dando pie a nuevas reflexiones sobre las dinámicas de reproducción social. 
Tardío y Terminal, debido a que la mayoría de las osamentas datan de la segunda mitad del primer milenio d.C. La movilidad residencial de la población tiene como efecto que, en el registro mortuorio, los portes locales están mezclados con aquellos de personas foráneas, quienes trajeron consigo la morfología cefálica que se les imprimió en su lugar de crianza. Es de notarse que, al contrario de lo esperado, no se han podido demostrar diferencias notables en las modificaciones de hombres y mujeres, o en personas de diferente inserción social, incluso entre aquellas poblaciones que exhibían formas variadas (Tiesler 2012). Todo parece indicar que los y las bebés recibían morfologías artificiales equivalentes de sus progenitores y que los jóvenes de la nobleza lucían una morfología cefálica acorde con el común denominador dentro de su localidad o región.

Aun por la aparente igualdad, el registro craneológico hace patente que en la mayoría de los territorios mayas se empleaban diversas técnicas de modelado cefálico, aspecto que encuentra su expresión en una variedad reiterativa de portes en cada localidad. Tal parece que las frecuencias de los aparatos destinados a la modificación cefálica estaban destinadas a permutar a través de la geografía cultural, como lo expresa el mapa de preferencias técnicas donde cambian las modalidades entre Belice al este y el corredor central petenero, donde se estilan proporciones similares en todas las colecciones que pudimos analizar. Esta distribución sugiere diversidad en morfologías cefálicas artificialmente logradas y a la vez uniformidad cultural dentro del corredor petenero central (Figura 2).

El área que rodea la cuenca media y baja del río Usumacinta se opone notablemente al patrón diversificado del hemisferio norte y oriental maya. Ahí se percibe un claro predominio de testas fuertemente inclinadas y tubulares, siendo este porte casi único en Palenque, Piedras Negras y Chinikihá durante el Clásico Tardío (Figura 3). Esta forma era el resultante de un procedimiento prolongado de compresión, efectuado con amarres circulares y tabletas ajustadas directamente sobre la testa infantil (Figura $4 a)^{4}$.

Río arriba, el Usumacinta recibe las aguas del río La Pasión. En su tiempo. la cuenca de este afluente propiciaba tierra fértil y ubicación estratégica para una serie de asentamientos mayas de larga ocupación, como son Altar de Sacrificios y Ceibal (Figura 4b). A estos centros se suma más tarde la ciudad de Cancuén, la cual fungia como puerta de entrada económica a las Tierras Altas mayas más al sur y hacia las montañas (Sharer y Traxler 2006a; Forné et al. 2009). De forma similar a sus vecinos río abajo, las personas que vivían en la cuenca del río La Pasión lucían una frente angosta y una disposición cefálica alargada y reclinada hacia atrás, aunque el cambio morfológico parece haber sido menos pronunciado entre ellos (Saul 1972; Quintani1la 2013; Tiesler 2014).

Al oeste del río Usumacinta y cuenca arriba del cauce del río La Pasión, las preferencias se transforman notablemente hacia las Tierras Altas, donde sus pobladores presentan testas anchas y cortas obtenidas tras la compresión en la cuna. Esta tendencia es evidente por ejemplo en el Clásico de Kaminaljuyú, o en Acul y Nebaj en el actual Departamento de El Quiché (Tiesler 2014; véase también Gervais 1989) (Figura 4c). T. Dale Stewart (1953: 296-97) confirma de manera similar el fuerte

\footnotetext{
4 Debemos tener en cuenta la idea de que, aparte de las formas cefálicas, también otros arreglos del cuerpo, como la representación nasal, siguen una distribución geográfica similar a la que trazan las formas artificiales de las cabezas (Kettunen 2005: 182-186).
} 


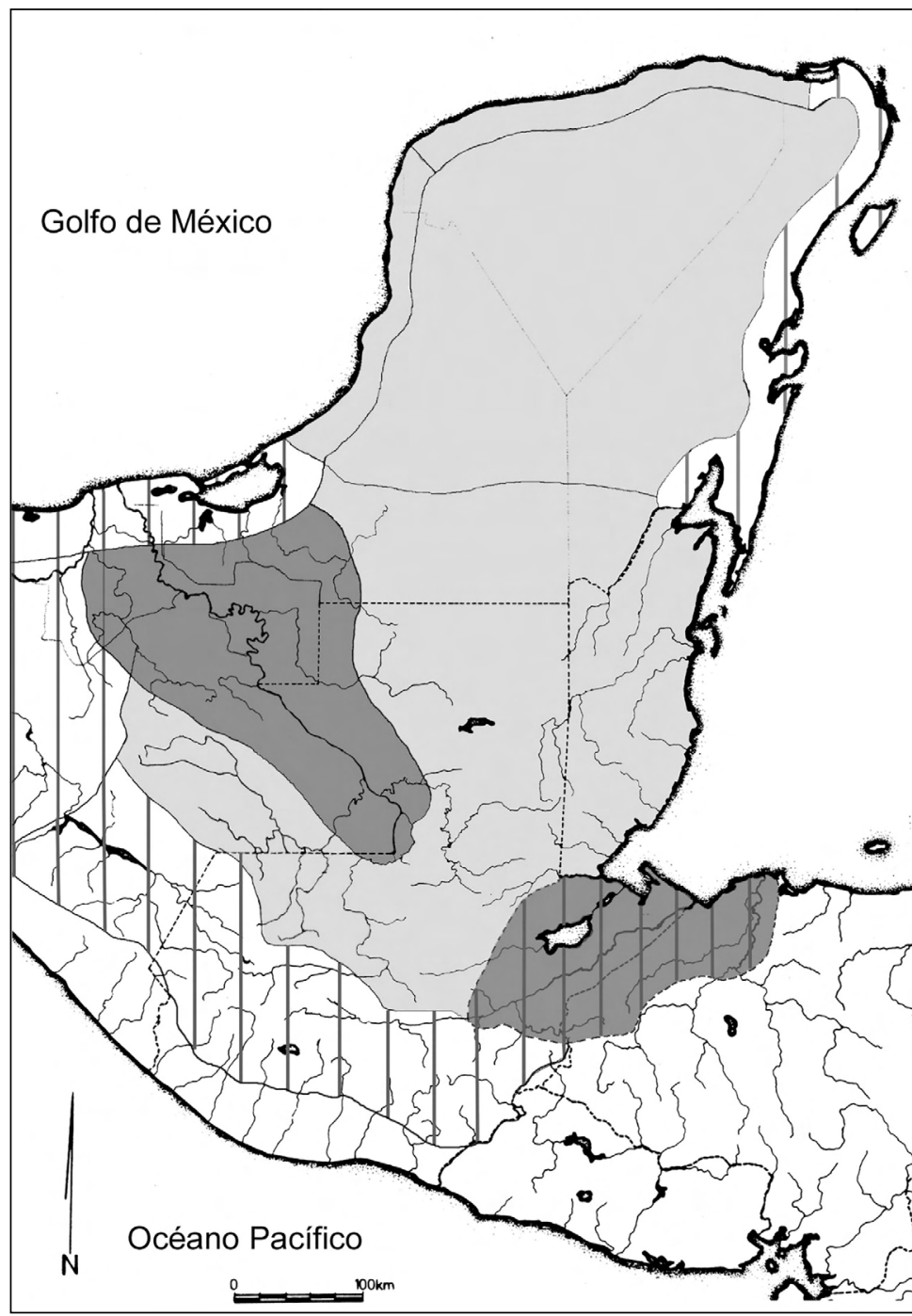

\begin{tabular}{r}
\hline$>70 \%$ tabulares oblicuos \\
\hline$\square 0-70 \%$ tabulares oblicuos \\
$<30 \%$ tabulares oblicuos
\end{tabular}

Figura 2. Distribución regional de formas cefálicas artificiales, documentadas en series esqueléticas mayas del periodo Clásico, donde se compara la proporción entre tabulares erectos y oblicuos. En las áreas marcadas con gris obscuro predominan testas angostas y reclinadas (tabulares oblicuas, logradas con amarres y tabletas cefálicas), en las áreas evidenciadas con gris claro prevalece una diversidad en formas y técnicas cefálicas y en aquellas rayadas predominan cabezas anchas y cortas, reproducidas con cunas compresoras (modificación tabular erecta). El caso de la periferia sureste es particular al mostrar las zonas rurales portes de cabeza anchos y cortos (tabulares erectos), mientras que la urbe de Copán concentra individuos con testa alargada y reclinada (tabular oblicua) (adaptación de Tiesler 2012, 2014). 


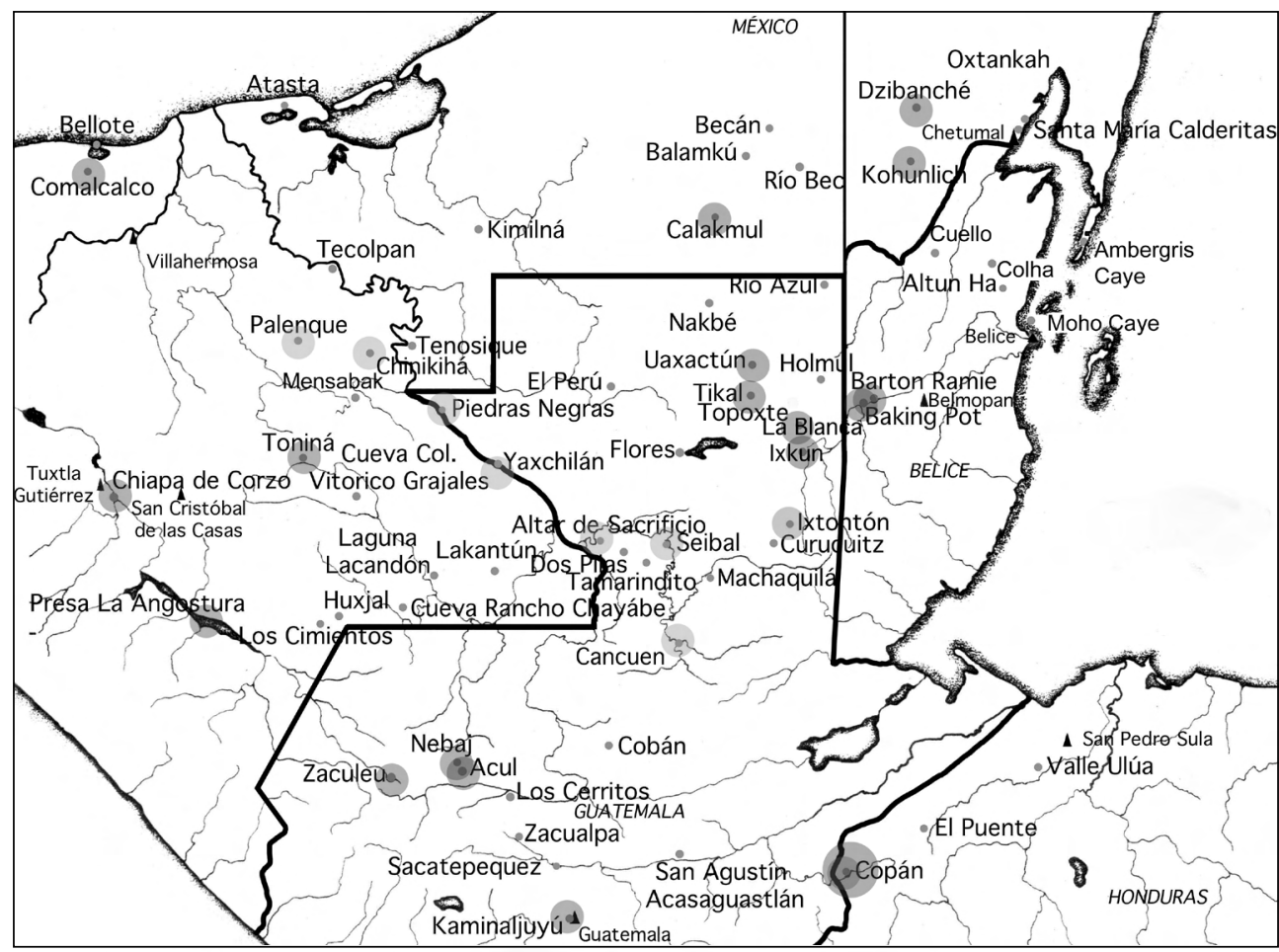

$>70 \%$ tabulares oblicuos

$30-70 \%$ tabulares oblicuos

$<30 \%$ tabulares oblicuos

Figura 3. Distribución de tipos cefálicos básicos documentados en series esqueléticas del periodo Clásico del centro y sur del Área Maya (incorporando información de Stewart 1953; Coe 1959; Haviland y Moholy-Nay 1992; Wright 1997; Tovalín et al. 1998; Wright y Witte 1998; Gómez 1999; Laporte 1999, 2005; Scherer et al. 1999; Tiesler 1999, 2012: 129-131, 2014, 2015b; Scherer y Wright 2001; Nuñez 2009, 2011; Quintanilla 2013; Scherer 2015; Chinchilla et al. 2015). Las series esqueléticas con muestra craneana preservada y cuantiosa que exhiben una fuerte predominancia de inclinación (tabular oblicua) aparecen en verde, en tanto que aquellas con predominio de acortamiento y ensanchamiento cefálico (tabular erecto) se marcan en azul. Las poblaciones locales con diversidad en portes cefálicos se marcan en color café.

predominio de las formas erectas entre los cráneos del periodo Clásico de Zaculeu (fases Atzan y Chinaq).

En los contextos fronterizos de relación intercultural, sobre todo en aquellos territorios localizados más al oeste del río Usumacinta y de reciente incorporación a las hegemónicas redes de dominio mayance, los portadores mayas de cabeza angosta e inclinada deben haberse destacado entre sus pares, sobre todo porque lucían una morfología cefálica opuesta: ensanchada y acortada. Un fenómeno análogo -la visible ostentación de la cabeza artificialmente modelada en contextos de pertenencias grupales opuestas- debe haberse dado también en otras zonas fronterizas que rodeaban los hemisferios del dominio maya durante el primer milenio. 

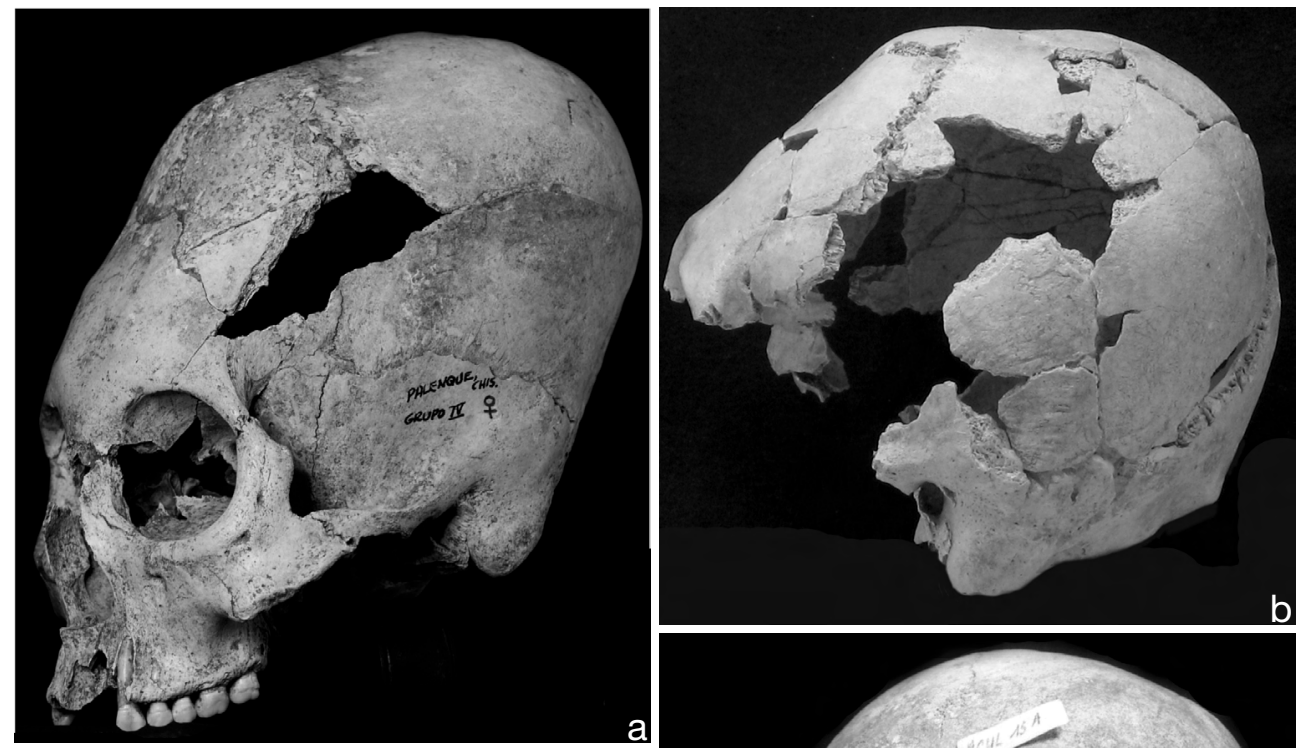

Figura 4. Formas cefálicas de diferentes partes del Área Maya: a) Cráneo femenino del Grupo IV, Palenque (OAF/INAH; fotografía de Vera Tiesler); b) Entierro 4-8 de Ceibal, Guatemala (968-38-20/N9876.2,

Peabody Museum of Archaeology and Ethnography, Harvard Museum. Copyright 2018 President and Fellows of Harvard College; fotografía de Vera Tiesler); c) Entierro $27 \mathrm{de}$ Acul/Nebaj, Guatemala (CEMCA/

IDAEH; fotografía de Vera Tiesler).

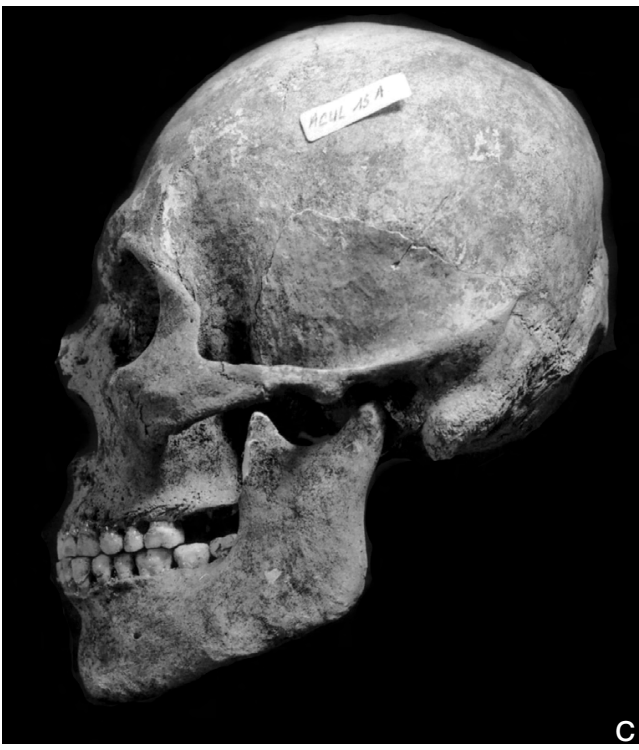

\section{De siluetas cefálicas mayas y lenguas vernáculas durante el Clásico}

Una de las características distintivas de la identidad cultural es el lenguaje hablado de un grupo, que exploraremos aquí entre las Tierras Bajas mayas, comparando la distribución de las lenguas vernáculas con las divisiones geográficas de las formas de cabeza reproducidas colectivamente. Ahora que el lenguaje jeroglífico maya se ha descifrado sustancialmente (Stuart 1987; Coe 1992; Houston et al. 2000), y desentrañado algunos aspectos gramaticales, parece probable que la gran mayoría de las inscripciones jeroglíficas se escribieron en un solo idioma asociado al ch'olano oriental (Houston et al. 2000). Este se convirtió en un idioma de prestigio, una especie de lengua franca para los escribanos que era similar al acadio utilizado en el Cercano Oriente, al latín en que los escribas medievales redactaban y el francés históricamente en uso entre la aristocracia europea. A pesar de la uniformidad lingüística del corpus escrito, en realidad 


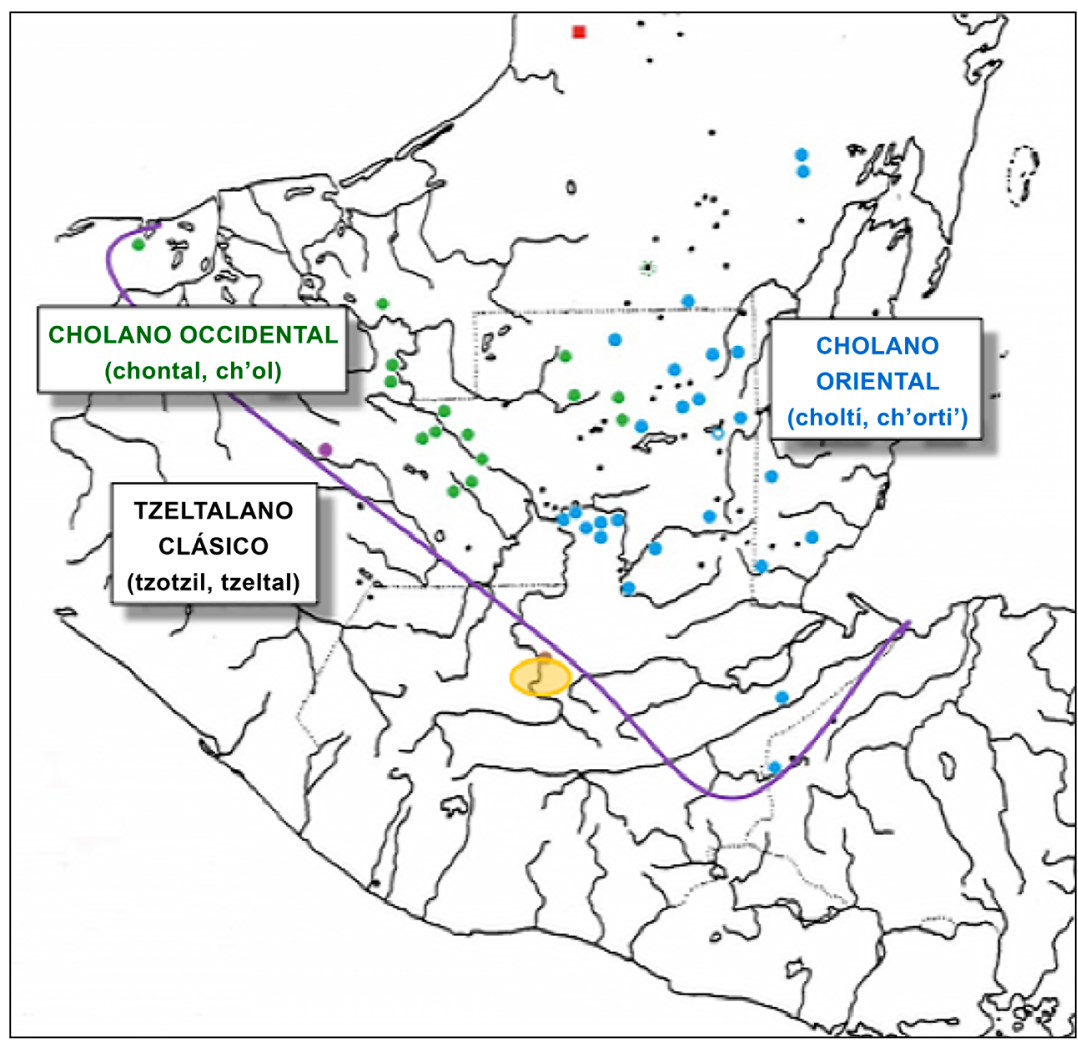

Figura 5. Distribución de las lenguas vernáculas en el hemisferio sur de los territorios mayances durante el periodo Clásico (actualizado de Lacadena y Wichman 2002).

coexistía con otras lenguas más populares en las Tierras Bajas mayas, algunas de ellas bastante diferentes del prestigioso ch'olano oriental. Especialmente relevante para este estudio es el hecho que algunos de los idiomas hablados hayan dejado su impronta en las mismas inscripciones del periodo Clásico. Bien aparecían como lenguas distintivas en las inscripciones para uso local o permeaban las demás inscripciones que eran redactadas en el idioma oficial a modo de inflexiones vernáculas. Aunque subliminales, estas inflexiones lingüísticas ponen al descubierto la lengua verdadera de su autor y, por lo tanto, la herencia cultural del escribano. Hay que tener en cuenta que cada uno de los idiomas regionales en uso estaba relacionado glotocronológicamente con idiomas mayas que todavía siguen hablándose hoy en día. Cuando se hablaban durante el primer milenio, se distinguían abiertamente del idioma oficial, no solo en fonología sino también en morfología y sintaxis (Lacadena 2000; Lacadena y Wichmann 2002, 2005; véase también Gronemeyer 2014a, 2014b).

De tal modo que, si ponemos en un mapa las isoglosas dialectales inscritas en los monumentos del área maya, surge una geografía dialectal coherente que muestra la distribución del lenguaje hablado en las Tierras Bajas mayas durante el periodo Clásico (Figura 5). Esta cartografía lingüística identifica los territorios donde el ch'olano oriental (un precursor de choltí y ch'orti modernos) no solo se escribía sino que se hablaba por el colectivo. Esta área corresponde al inmenso corredor que 
representa el hemisferio oriental de las Tierras Bajas mayas, incluyendo grandes sectores del Petén y las cuencas de los ríos Motagua y La Pasión, así como la parte sur del estado de Quintana Roo hacia el norte. Su extensión regional bordea una segunda área dialectal que se identifica con el ch'olano occidental (un precursor de chontal y ch'ol modernos). Esa lengua se hablaba en el Petén occidental y a lo largo de toda la cuenca del Usumacinta. Al oeste, los ch'olanos occidentales bordeaban una tercera zona dialéctica, identificada con el idioma tzeltalano (afiliado al tzeltal moderno y el tzotzil), que se pronunciaba entre los habitantes de Toniná y sus alrededores y también se escuchaba en las Tierras Altas chiapanecas hacia Comitán.

Finalmente, los lugareños de la zona centro y norte de Yucatán conformaban una cuarta zona dialectal, identificada con el yucatecano (un precursor de yucateco, lacandon, itzaj y mopan modernos). En una comunicación aún no publicada, Albert Davletshin y Dmitri Beliaev han reunido recientemente pruebas que respaldan la existencia de lo que podría haber sido una quinta área lingüística. Han identificado una lengua antigua, potencialmente afiliada al k'iche', que se ha perpetuado en las inscripciones de cerámica de Chama y Nebaj de las Tierras Altas de Guatemala, justo al sur de los hablantes de las Tierras Bajas de los hablantes del ch'olano.

Aunque quizás no sorprenda, cabe destacar algunos patrones emergentes que detectamos cuando comparamos la distribución geográfica de las formas cefálicas culturalmente reproducidas y la correspondiente al lenguaje de sus portadores. Estas se sobreponen en algunas partes y en otras coinciden (Figuras 2 y 5). Por ejemplo, las diferencias en el lenguaje hablado y la preferencia colectiva en el porte cefálico demarcan un límite geográfico a lo largo de las laderas de Chiapas. Esta fue la división cultural entre grupos lingüísticos mixe zoque ismeños, los dialectos ch'olanos (Usumacinta) y los del chontal que se escuchaban en las llanuras costeras hacia Tabasco y Veracruz (Figura 5) (Justeson et al. 1985: 68-70; Lacadena y Wichmann 2002, 2005; Mora Marín et al. 2009; Pallán 2009). Desde tiempos inmemoriales, este corredor mesoamericano ha marcado una intersección de diferentes pueblos con distintos orígenes culturales y diferentes dominios políticos y redes comerciales, un crisol que ganó prominencia durante la segunda mitad del periodo Clásico (Vargas 2001; Wyllie 2002).

Curiosamente, la demarcación lingüística entre los territorios con idiomas mayas y no mayas sigue aproximadamente aquella de preferencias discrepantes en morflogía cefálica, confirmando la connotación «étnica» que una vez tuvo la modificación cefálica, y confirma su potencial analítico para rastrear las dinámicas socioculturales y poblacionales que en un tiempo fundamentaban la división. En tanto que las cabezas estrechas, inclinadas y alargadas se muestran prominentemente entre los mayas occidentales ch'olanos de la cuenca del Usumacinta, la mayoría de las poblaciones no mayas limítrofes, del habla tzeltalana y zoque, lucían visiblemente cabezas anchas y acortadas. Más al sur, los montañeses de Nebaj y Acul, con aspecto cefálico también ancho y corto, hablaban una variante del idioma k'iche'.

Las diferencias en la morfología craneal entre los hablantes de diferentes lenguas mayas y no mayas deben haber sido más evidentes más allá y dentro del valle del Usumacinta. Ahí, la apretada y prolongada constricción de la cabeza de los lugareños hacía resaltar no solo la parte trasera de su testa sino también su fisonomía facial (Tiesler 2014: 46-48). Los amarres cefálicos propiciaban el prognatismo facial, identificado por la prominencia de las facciones nasales, de los labios y pómulos (Figura 6a). En ocasiones el mismo empuje compresor podía borrar la muesca de la raíz nasal entre las cejas, y así la nariz parecía emerger directamente desde debajo de 
la frente. Aparte de los cambios propios de la morfología cefálica debe haber habido también cambios posturales en la persona viva. La redistribución del peso cefálico, junto con una mayor inclinación de la base craneana, hacía que los individuos con cabeza severamente alargada alzaran su mentón y parecían mirar hacia abajo con los párpados semi-cerrados. Las convenciones de retrato entre los mayas del Clásico, aún exageraban estas características morfológicas y posturales en los retratos, por lo cual podemos pensar que los consideraban sumamente deseables y dotados de belleza (Sánchez 2008; Tiesler 2014: 48).

Tal aspecto facial y las mismas fisonomías inclinadas y angostas de la bóveda, habrán sido fácilmente reconocibles también entre las poblaciones que poblaban la franja de contacto y tránsito, la mayor parte de ellas hablantes del ch'olano occidental, oriundos de lugares tales como Palenque, Jonuta, Pomoná, Piedras Negras, Yaxchilán y Bonampak (Figuras 6a y 6b). Notablemente, los vecinos al oeste y sur de Palenque, como aquellos de Toniná y Chiapa de Corzo (Figura 6c), no habrían compartido su aspecto físico. Nuestros registros craneológicos (Tiesler 2010) sostienen que los lugareños de Chiapa de Corzo reproducían testas tabulares erectas desde inicios del periodo Clásico, mientras que, a cierta distancia de Palenque, los habitantes de Toniná eran feroces opositores políticos y militares. Algunos estudiosos sostienen que Toniná fue habitada no por hablantes del ch'olano occidental sino por otra comunidad linguística no maya (Ayala 2002). Los restos esqueléticos del sitio central y de sus aldeas satélites fueron explorados por la Misión Francesa (Centro de Estudios Mexicanos y Centroamericanos en la Ciudad de Guatemala) durante la década de 1970 para ser analizados por primera vez por Arturo Romano (1979; Romano y Jaén 1990; véase también Tiesler 1998, 1999). En contraste con Palenque, ahí predominaban los portes cefálicos anchos y cortos. Este aspecto parece haberse resaltado aún más, al constreñir la bóveda infantil con una correa divisora que resaltaba, en forma visible, ambos lóbulos laterales. Este contrastante estilo de modificación craneana ancho y plano habría sido inmediatamente reconocido como no ch'olano occidental por los mayas del Usumacinta, una declaración incorporada negando cualquier raíz occidental de ch'olano maya. Esta impresión también la dan las representaciones artísticas nativas.

Aunque la iconografía maya clásica siguió sus propias convenciones artísticas, es una suerte para el escrutinio académico que sus representaciones de la apariencia física humana sean más realistas y más detalladas que otras tradiciones iconográficas americanas, incluidas la mayoría de las convenciones artísticas mesoamericanas. Con respecto a la forma de la cabeza, los escultores de los paneles y las estelas de Toniná, los cuales suponemos eran hablantes de maya tzeltalano, representaban sus dinastías locales con perfiles craneales tanto altos como cortos (ver Figura 6c). Por el contrario, sus humillados cautivos mayas de Palenque y otras comunidades del Usumacinta, hablantes del ch'olano, son retratados con cabeza inclinada y frente alargada hacia atrás (ver Figura 6b), coincidiendo con las formas cefálicas tubulares de sus registros craneales (Tovalín et al. 1998; Scherer 2006, 2007, 2008, 2015; Tiesler 2012). Los cautivos de otros reinos conquistados, como Calakmul, con un repertorio más diversificado de morfologías de cabezas artificiales, lucían siluetas de cabezas diversas y menos pronunciadas. Las distinciones en formas de cabeza representadas por los artistas de Toniná aparentemente se hacen eco de lo local (tzeltalano) frente a los antagónicos extranjeros (ch'olano occidental). Esta demarcación por sí misma parece indicar que la forma de la cabeza era un elemento cultural distintivo, que, fácilmente visible, estaba imbuido de un significado diferenciado, identitario y étnico. 


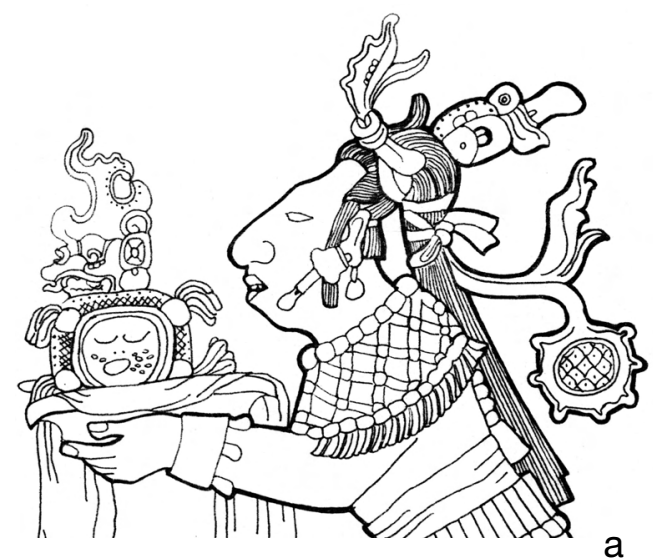

Figura 6. a) Fisonomía facial de la Señora Tz'ak B'u Ahaw, tal como se retrata en la Tableta del Palacio, Palenque, Chiapas. El semblante de la que en vida fue la esposa del gobernante Janaab' Pakal's, se retrata con una exagerada inclinación de la frente, que además muestra un contorno cóncavo. Resaltan las facciones faciales protruyentes de su cara, más allá de lo que muestra su cráneo y lo que en todo caso pudiera ser factible anatómicamente (trazo de Mirna Sánchez); b) cautivo de Palenque, tal como se retrata en una escena esculpida en el sitio rival de Toniná, mostrando frente sumida y severamente inclinada junto con bóveda alargada (trazo de Simon Martin); c) Dignatario (ajk'uhu'n, 'sacerdote'), hablante del tzeltalan y lugareño de Toniná (Monumento 183), mostrando una silueta cefálica alta y acortada (trazo de Simon Martin).
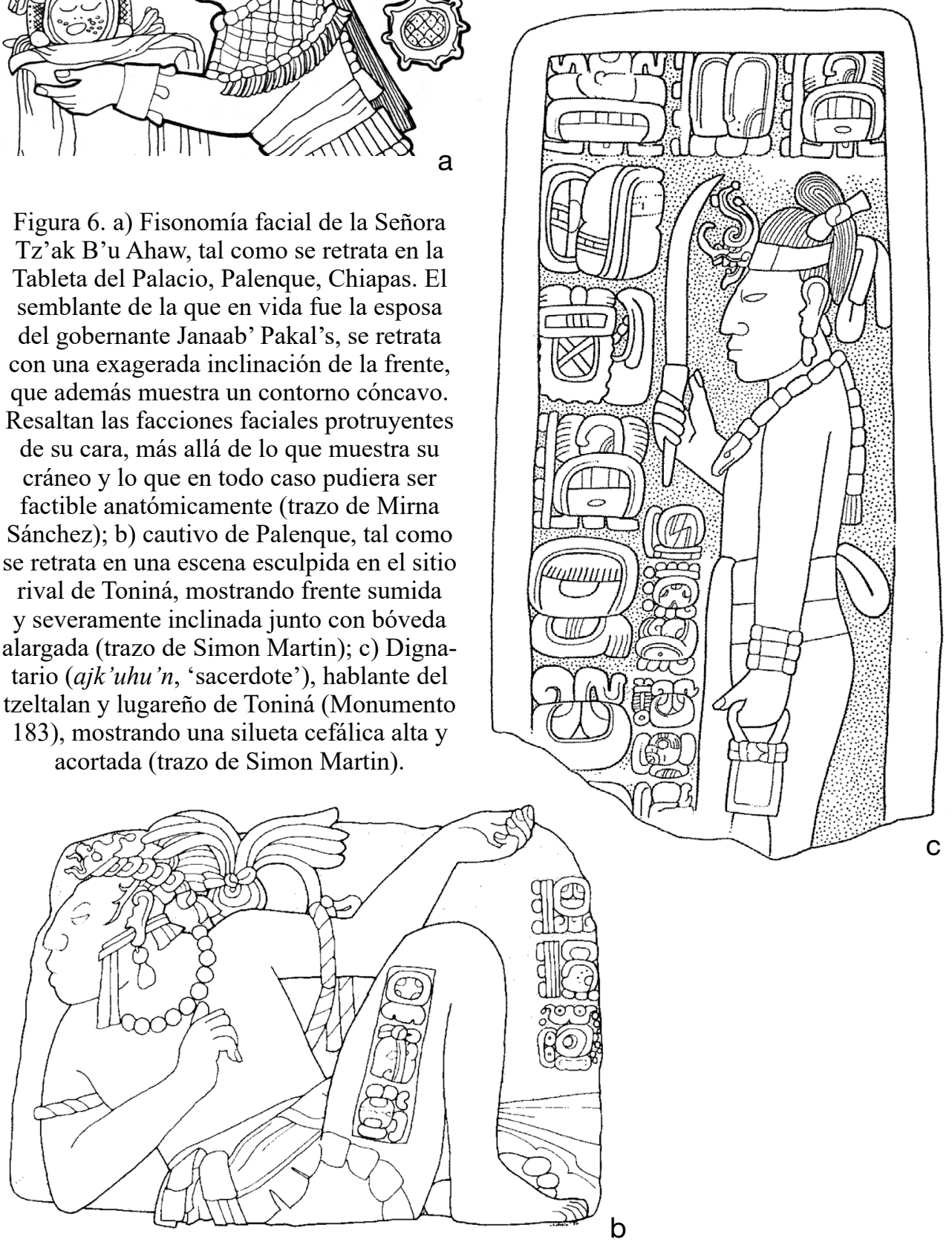
A una escala más regional, también se deduce que la preferencia de la forma de la cabeza correspondió en cierta medida con la afiliación lingüística, tal como lo hemos descrito anteriormente. Durante el Clásico Tardío, formas dialectales propias del ch'olano occidental, algunas de las cuales tienen lugar en los alrededores de Palenque, comenzaban a ser utilizadas en los textos de las áreas al este, donde el ch'olano fue hablado tradicionalmente. Estos cambios de lenguaje se extienden gradualmente fuera de la cuenca del río Usumacinta, a través de la cuenca del río La Pasión y hacia el lejano río Motagua en el borde sureste de las Tierras Bajas mayas (Lacadena y Wichmann 2002; Hruby y Child 2004). Posiblemente, la adopción de estas formas lingüísticas fue consecuencia del prestigio y el poder político que emanaban las entidades occidentales dentro de la región.

En la misma Toniná, los escribas locales redactaron sus textos en su propio dialecto peculiar, aferrándose a las convenciones escritas del ch'olano oriental y no aceptando nunca, sino de hecho resistiendo, las introducciones dialectales de los ch'olanos occidentales. Muy posiblemente, la resistencia entre los cortesanos de Toniná para adoptar el nuevo lenguaje de moda fue alentado por su identificación del ch'olano occidental con la lengua de Palenque, enemigo ancestral de Toniná. Es evidente, por lo tanto, que el antagonismo entre Palenque y Toniná se manifestó no solo en la lucha política entre dos reinos en competencia sino, más allá de la arena política, fue un choque entre dos grupos étnicos en los que la apariencia visiblemente distinta de cabezas y los tonos disonantes de diferentes lenguas jugaron un papel importante.

\section{Cambios versus permanencia tras el ocaso de las hegemonías mayas}

El final del periodo Clásico en las Tierras Bajas mayas estuvo marcado por un panorama político cada vez más conflictivo y separatista. Redadas, guerras y tomas de cautivos dominaban los siglos VIII y IX mucho más que los anteriores, una tendencia notable tanto en la iconografía como en los registros arqueológicos del centro y sur de las Tierras Bajas (Webster 2002; Barrett y Scherer 2005; Martin y Grube 2008; Tiesler y Cucina 2010, 2012; Tiesler 2015a). En este hostil clima político, la formación de alianzas y antagonismos en los intercambios políticos merece una mayor consideración.

Como ayuda visual hemos superpuesto nuestras cartografías vernáculas y cefálicas con el esquema de contactos y redes políticas entre los reinos mayas clásicos, elaborado por Simon Martin y Nikolai Grube (2008: 21) (Figuras 7a y 7b). Por lo pronto

Figura 7 (página opuesta). Esquema de redes políticas entre los reinos mayas del periodo Clásico (adaptado de Martín y Grube 2008: 21): a) Sobreposición de la distribución de lenguas vernáculas mayas que se resaltan en gris (véase también la Figura 5): gris obscuro: tzeltalan; gris claro: ch'olano occidental; gris medio: ch'olano oriental; b) Sobreposición de la distribución de portes cefálicos evaluados en las series esqueléticas de los mismos sitios (véase también la Figura 3) (Tiesler 2012: 129-31): la preferencia por formas inclinadas (tabular oblicua) se señala en gris claro; poblaciones locales mostrando diversidad en formas cefálicas se marcan en gris medio y otras con franco predominio de portes tabulares erectos aparecen en gris obscuro. Nótese que la población del núcleo copaneco (anillo interior) muestra formas cefálicas predominantemente oblicuas, mientras que las poblaciones enterradas en su periferia y en las afueras del Valle de Copán muestran una proporción predominante de formas erectas (anillo externo). 

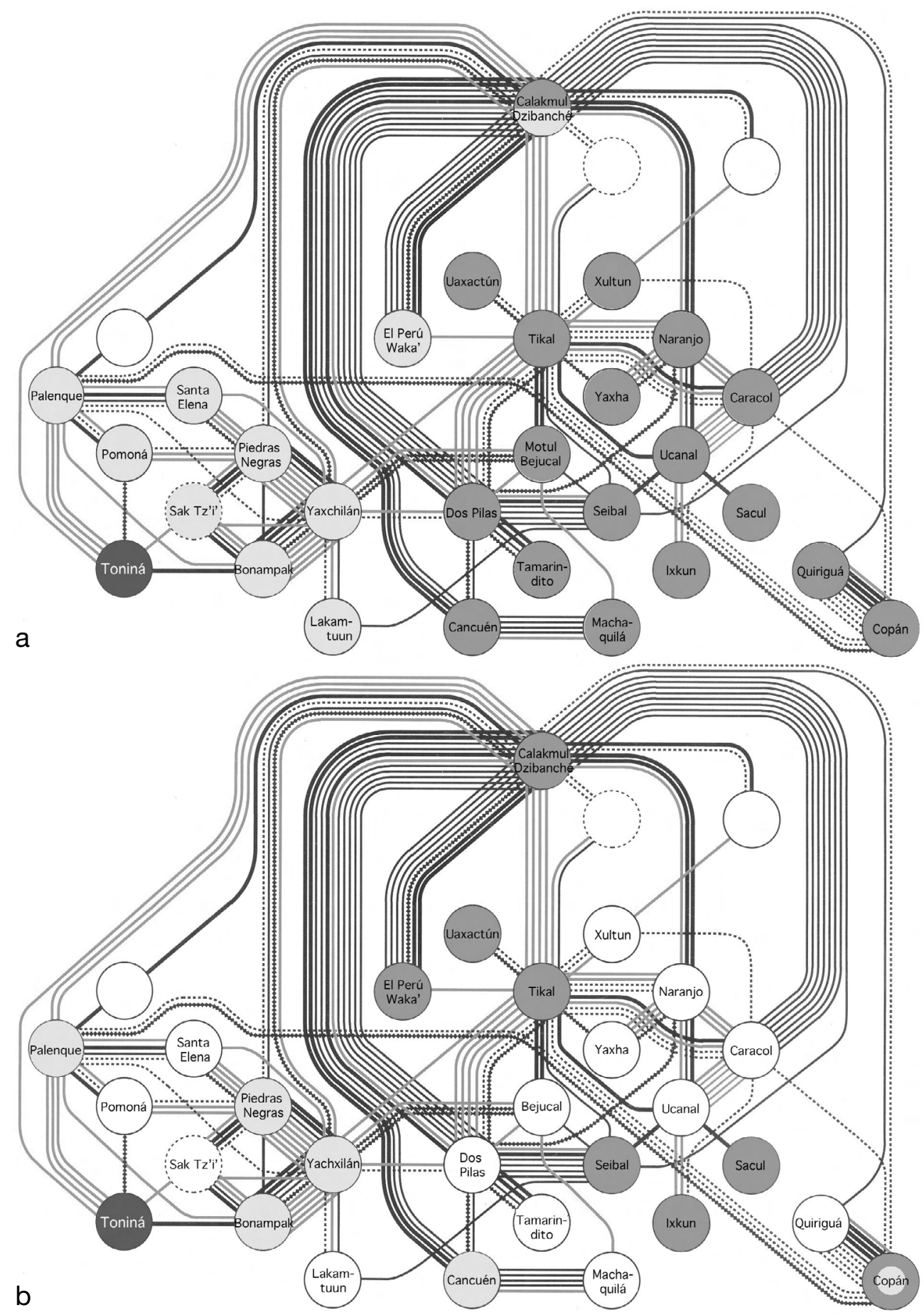
hemos supuesto que se hablaba ch'olano oriental por un lado y ch'olano occidental por el otro, más el tzeltalano en Toniná. Al menos visualmente, los intercambios parecen ser más abundantes entre los reinos de cada uno de los dominios lingüísticos, que parece separar los sitios marcados en gris claro (ch'olano occidental) de otros marcados en gris obscuro (ch'olano oriental) (Figura 7a). Las menciones en las inscripciones de la jerarquía, los contactos diplomáticos, los conflictos, los lazos familiares y otras relaciones entre las dos esferas son menos frecuentes que dentro de cada esfera lingüística.

Aun así, las redes entre grupos de una misma glota no estaban exentas de conflictos internos y de guerras, tal como subraya el mismo esquema. En este panorama, el Reino de las Serpientes de Calakmul, al norte de Petén, presenta un ejemplo interesante, ya que parece haber adoptado el ch'olano occidental según el cambio de inflexiones en las inscripciones locales. En algún lugar entre las lenguas del este y occidente de Petén se encuentra también la lengua hablada en El Perú Waka', claramente afiliado en ese momento al reino de Calakmul, no a otros reinos de ch'olano occidental. El conflicto y las batallas frecuentes parecen redefinir las relaciones ulteriores entre los centros de habla ch'olano occidental y Calakmul, y aún más agudamente, entre ch'olanos occidentales y tzeltalanos de Toniná.

Menos seguras que las atribuciones lingüísticas de las ciudades clásicas mayas son las preferencias por alguna modificación cefálica específica entre aquellos colonos que vivieron y murieron en los centros documentados por los epigrafistas. Pero incluso aunque la investigación es incompleta, sugiere tendencias en la conformación de la cabeza que, una vez más, siguen las directrices lingüísticas entre los reinos mayas clásicos, como se ilustra esquemáticamente por Martin y Grube (2008: 21) (Figura 7b). Su superposición en el diagrama de interacción hegemónica establece una vez más que los reinos occidentales, donde predominaba el alargamiento cefálico, se apartan del resto de las comunidades del Petén central durante el Clásico, donde prevalece la diversidad en los portes cefálicos. La población tzeltalana de Toniná (coloreada más obscuro) se diferencia por usar predominantemente cabezas cortas y anchas.

Una segunda tendencia en esta superposición atañe a las formas de la cabeza de las comunidades ch'olanas que se alinean en las fronteras sureñas hacia las Tierras Altas. Contrario a lo esperado por su asociación vernacular, los lugareños de Cancuén lucían una testa artificialmente inclinada (Quintanilla 2013). Más compleja todavía es la distribución en la franja sudeste de los reinos mayas, rodeados de poblaciones no mayas de ascendencia centroamericana. Las poblaciones multiculturales que fallecían (y presumiblemente vivían) en diferentes sectores del valle de Copán, Honduras (Tiesler y Cucina 2010; Tiesler 2014: 215-219; Suzuki 2018) durante el Clásico Tardío hablan de una separación. La valoración de doscientos cráneos del centro de Copán y sus alrededores parece comunicar que los pobladores que vivían en las vecindades de su núcleo urbano (culturalmente y «étnicamente» maya) lucían la frente y testa angosta e inclinada, mientras que más de la mitad de sus vecinos en las periferias la mostraban ancha y corta. Al parecer, no solo la afiliación lingüística en sí, sino también la ubicación en relación con otras poblaciones debe haber influido en la tradición colectiva de modelar la cabeza, apoyando la idea de que -en este contexto- las modificaciones craneales reproducían las particularidades de la cultura (Sharer y Traxler 2006b).

Consideradas en conjunto, las divisiones entre las lenguas habladas y las formas craneanas, permiten vislumbrar con una nueva óptica las redes de intercambio y demás enlaces entre los reinos de las Tierras Bajas mayas antes del colapso (Martin y Grube 
2008) (Figura 7). Las historias grabadas en piedra, al denotar estratégicos enlaces matrimoniales, alianzas y enemistades, tributos y dependencias, trazan un devenir histórico mucho más inestable y conflictivo que aquel de las lenguas vernáculas y los portes cefálicos, y no excluyen hablantes de un mismo idioma: se libraban batallas constantes entre grupos que habían cooperado previamente en redes ch'olanas occidentales hacia el amargo final del periodo Clásico. Compartir la misma lengua hablada o, para el caso, la misma forma de cabeza, ya no facilitaba alianzas duraderas entre las aristocracias de una misma zona, como serían los habitantes de Yaxchilán, Palenque o Piedras Negras, cuyos mandos estuvieron en guerra hasta el final de su gestión. Lo mismo puede decirse, por supuesto, sobre Copán y Quirigua, o para Naranjo y Caracol, al este, cuyas poblaciones, presumimos, eran hablantes del ch'olano oriental.

A medida que avanzamos hacia el cierre del primer milenio d.C. el creciente clima de agitación política plantea interrogantes sobre los elementos de ruptura y de persistencia cultural durante y después del colapso

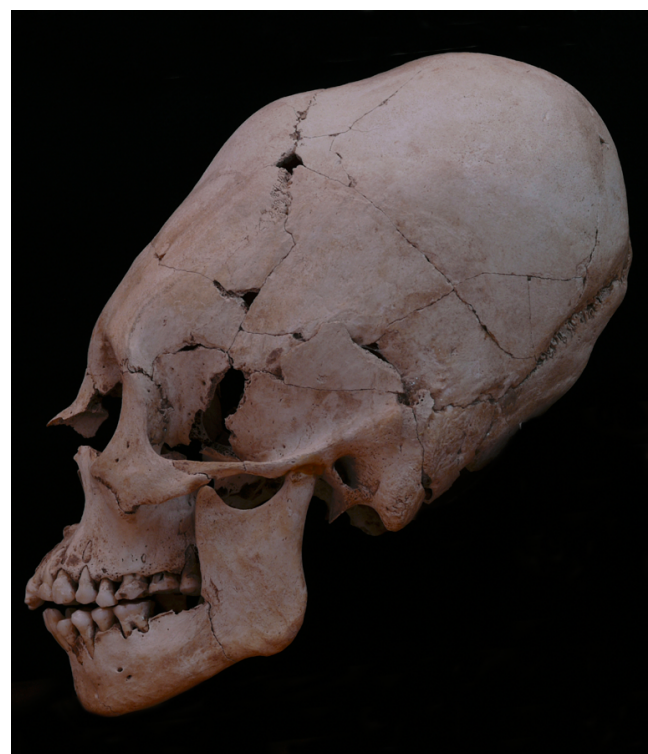

Figura 8. Cráneo fechado para la época posterior al colapso, bordeando las Tierras Altas al sur. Aún muestra una morfología artificial

al modo del Clásico, al tener una bóveda angosta, alargada e inclinada hacia atrás (tabular oblicuo, variante pseudo-anular): Entierro 60, Altar de Sacrificios, Guatemala (Saul 1972; Fase Jimba [900-950 d.C.]; Peabody Museum of Archaeology and Ethnography, Harvard Museum. Copyright 2018 President and Fellows of Harvard College; fotografía de Vera Tiesler). maya. El escenario combinado que surge de la lengua, la formación de la cabeza y las redes políticas identifica algunas dinámicas de sustitución cultural y permanencia mediante la identificación de aquellos elementos culturales y dinámicos que fueron retenidos y otros que fueron reemplazados tras la ruptura institucional y social maya.

Si bien la epigrafía guarda silencio sobre los últimos discursos de la élite de las Tierras Bajas mayas clásicas, antes de consumarse la destrucción y el abandono de las cortes en los núcleos urbanos, el registro craneano es más elocuente: la información que comunican las series de bóvedas craneanas mayas fechadas para el siglo IX en adelante, apoya la idea de una continuidad en las preferencias entre la gente del común, subrayando, una vez más, la dimensión equitativa y conservadora de la práctica y su calidad transgeneracional y femenina. Parece que generaciones después de que los miembros de las élites dinásticas habían perecido o salido con sus familias para siempre, las poblaciones mayas remanentes seguían conservando sus formas ancestrales de moldear las cabezas de sus bebés. De hecho, parece haber continuidad en los procedimientos de configuración cefálica hasta el final, como hemos identificado en otros trabajos entre las últimas generaciones de colonos en Ceibal y Altar de Sacrificios en el área del río La Pasión (Tiesler 2015a) (Figura 8). 
Puesto en contexto con los territorios de habla ch'olana oriental, la permanencia de la práctica cefálica refleja una vez más la continuidad en el lenguaje hablado en la esquina suroeste de las Tierras Bajas mayas. Las fuentes etnohistóricas indican que esta área aún seguía habitada por hablantes de ch'olano hasta el siglo XVI (Mora Marín et al. 2009). En una nueva era mesoamericana, con sus nuevos emblemas sacros y orientaciones cosmológicas, damos por seguro que las anacrónicas formas oblicuas debieron adquirir nuevos significados para sus portadores y para aquellas personas que interactuaban con ellos.

\section{De modificaciones cefálicas y mitos de creación humana}

Hemos explorado a lo largo de este documento las correlaciones geográficas y culturales entre los modelados cefálicos, las lenguas habladas y las redes políticas. Al examinar estas relaciones, podemos reconocer cómo la modificación craneana desempeñaba un papel activo en la reproducción de la identidad y social colectiva, a la vez que en las ciudades fronterizas también podía significar otredad y antagonismo. Puesto en contexto con nuestros enunciados introductorios sobre etnicidad, ambas dinámicas se muestran como expresiones ancestrales, de pertenencia grupal y propiamente «étnicas». En lo que considera la variación visible de las morfologías, pensamos que era apta para anunciar abiertamente la afiliación y distinción de su portador vivo o incluso sacro. El panteón de entidades divinas puede haber proporcionado modelos para orientar la impresión de las siluetas cefálicas, aunque la relación no puede demostrarse de modo concluyente (Taube 2003; Houston et al. 2006; Tiesler 2010, 2014, 2015a; García Barrios y Tiesler 2011). Aun así, vale la pena detenernos en la discusión de algunos patrones compartidos entre las morfologías cefálicas en la imaginaria sacra antropomorfa y la histórica real de los humanos: al menos en las Tierras Bajas mayas, los retratos antropomorfos del periodo Clásico tienden a representar deidades jóvenes, como el dios del maíz, con la frente fuertemente inclinada y la bóveda craneana alargada hacia atrás (Taube 2003). Algunos autores también han propuesto que la cabeza inclinada se parece a la cabeza de un jaguar (Sotelo y Valverde 1992). A la inversa, las deidades viejas, como Chaahk y el Dios L, suelen ser representados con cabezas achatadas en sus versiones antropomorfas. Los moradores sacros del inframundo, como el Dios A y el Dios N, se encuentran entre las pocas entidades que se representan con un occipucio redondeado (García Barrios y Tiesler 2011).

No obstante los potenciales motivos sacros y sus diversificados resultados, queda palpable que los tratamientos cefálicos eran costumbres que se realizaban de forma inclusiva en los hogares. Aunque el resultado morfológico quedaría inscrito permanentemente en el semblante y era visible en público, en realidad se generaba en el «interior» de la casa. Las maniobras técnicas no distinguían entre descendientes masculinos y femeninos. Tampoco las formas resultantes parecían haber sido distintivo de ningún sector social ni reino en las Tierras Bajas, si nos atenemos al registro esquelético y los retratos del área (Cetina et al. 2018). Estas reflexiones resaltan los puntos ya señalados por William Duncan (2009: 187-88), quien habla de las maniobras sobre la cabeza como una medida protectora y de «incorporación». En el curso del ejercicio diario de los procedimientos pertinentes, las parientes recurrieron a la compresión, la envoltura, al vendaje o al masaje de la cabecita hasta que el cambio 
se convertiría en permanente y hasta que las fontanelas habrían «secado» (Duncan 2009: 187-88; Duncan y Hofling 2011). Tras el contacto europeo, los mayas yucatecos todavía se referían a este proceso como up' $k$ 'abtah, traducido como «enderezar la cabeza del recién nacido para enmendarlo o adaptarlo» (Barrera Vásquez 1980: 901).

Hay dos explicaciones potenciales para tal, aparente, igualdad social. En primer lugar, e independientemente de que fuese bebé varón o hembra, las practicantes fueron con toda probablilidad siempre mujeres, cuidadoras de segunda o tercera generación, quienes modelaban las cabezas de sus recién nacidos, aún sin voluntad propia (Pérez 2018). Esta condición se refuerza a sabiendas que los procedimientos caían en una época de socialización incipiente, en la que el pequeño todavía era considerado «un fruto inmaduro», una «no persona» a la que se tenía que preparar para su ulterior integración social (Cervera 2007; Tiesler 2011; Scherer 2018). Era una etapa de existencia pre-humana, previa a la integración social, previa a que «el pensamiento» entrara en el cuerpo del pequeño. La integración comunal tenía lugar normalmente a una mayor edad y se consagraba con ceremonias de tránsito, como la asignación de nombres, cortes de pelo y rituales de iniciación propiamente de juventud.

Aunque las practicas cefálicas hayan sido inclusivas (o justamente por ello mismo), sin duda estaban impregnadas por un profundo pensamiento sobre el cuerpo y lo humano. Una serie de representaciones en la cerámica del Clásico maya son reveladoras del acto de modelar con dedos y espátulas ciertos objetos mantenidos en las manos. Por los contextos y los atributos iconográficos suponemos que los personajes son dioses, pero, en cambio, los objetos modelados son cabezas humanas. Los textos jeroglíficos que acompañan las escenas, aunque breves, son claros en su contenido: «[ellos] dieron forma a los seis primeros hombres»o «Los primeros seis hombres fueron formados. La clave es el verbo pak', que significa «dar forma a algo con las manos», tal como podría ser un ladrillo o la arcilla (Beliaev y Davletshin 2014).

Muy probablemente, estas representaciones recogen partes de mitos del periodo Clásico sobre la creación de una de las dos razas humanas que fueron modeladas, tal como se menciona en el Popol Vuh: aquellas confeccionadas en barro o aquellas hechas de maíz. ¿Pero cuál de las dos? En una de las escenas, reconocemos sentado a la derecha a ltzam Kokaaj, uno de los dioses supremos del panteón clásico maya (Figura 9). Frente a él, se retrata uno de los dos dioses artesanos (con cara de simio), Hun Chwen o Hun Batz y, detrás de él, se reconoce al dios del maíz gracias a la planta que emerge de su cabeza. La presencia del maíz en la conformación de estos seis primeros hombres nos permite relacionar esta escena con la última creación, es decir, aquella exitosa tarea de formar al hombre gracias al uso de la masa de maíz. De hecho, las cabezas humanas modeladas en la escena pueden identificarse claramente como mayas del Clásico. Mirando de cerca, los modelados muestran diferentes aplanamientos. Históricamente, fueron más del $70 \%$ las personas mayas quienes durante el Clásico lucieron una morfología cefálica culturalmente modificada (Tiesler 2012, 2014). Cuando un individuo maya clásico imaginaba la última creación humana, debe haber pensado en una imagen de sí mismo (véase también López Austin 2009).

La idea de que el arte sacro del modelado de los primeros hombres involucrara a más de una deidad plantea además preguntas sobre la variedad de la apariencia, incluidos los roles de cabeza corta, sin contorno artificial, o lo que los mayas llamaban cabezas «redondas». ¿Fue a propósito o fue el descuido lo que llevó a algunas madres a dejar la testa de sus hijos al natural? Esta conjetura está justificada, dado 


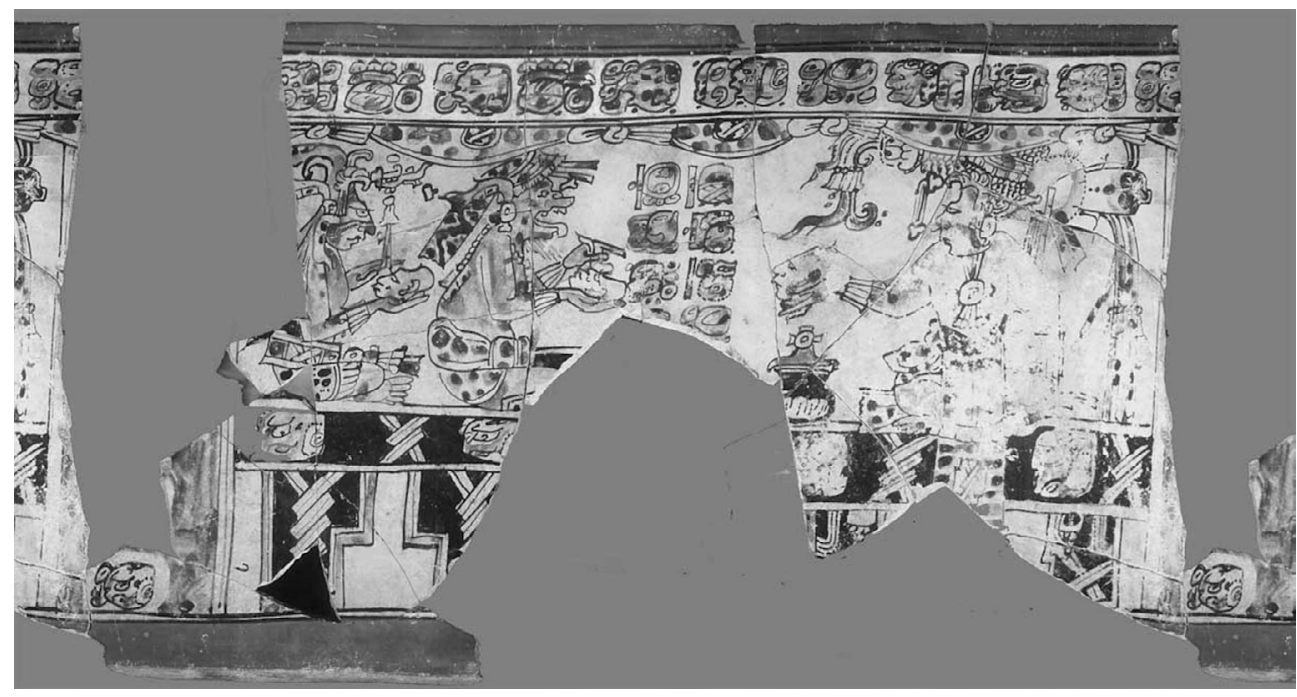

Figura 9. Escena sobrenatural donde un dios supremo del panteón clásico maya, ltzam Kokaaj, está sentado a la derecha, mirando a Hun Chwen o Hun Batz', representado con semblante de simio. A la izquierda, el dios del maíz participa con las otras deidades en el formado de cabezas humanas, como parte de la última creación exitosa de la humanidad. Se utiliza masa de maíz para constituir los cuerpos. El texto se lee como «u-pa-k'a-wa WAK-YAX-WINIK UH-ti-ya HO'-B'AL-NAL» (upak'a'w Wak Yax Winik uhtiiy Ha' $B^{\prime} a$ '/ $\left.N a /\right)$ : «ellos formaron las seis primeras personas; ocurrió en el lugar de las cinco flores» (Archivo Kerr, K8457).

que, en el área maya del periodo Clásico, sólo una pequeña proporción de las series esqueléticas se presenta sin los efectos visibles de la modificación craneal. Solo de occidente a oriente se percibe que el porcentaje de cráneos no modificados va proporcionalmente en aumento, llegando a cifras notables entre los mayas beliceños. Más allá de las tendencias costumbristas y dada la evidente falta de reglamentación en la práctica modeladora, podría ser el descuido materno o los problemas de salud (secundarios al proceso de compresión), los que podrían haber llevado a las madres a omitir o terminar los procedimientos de la cabeza antes de que dejaran huella permanente, tal y como hemos postulado en otro trabajo (Tiesler 2014: 260-262).

\section{Conclusiones}

Concluimos nuestro recorrido a dos voces con la idea argumentada de que tanto el habla como el semblante deben haber «incorporado» identidad, pertenencia grupal y etnicidad, misma que se comunicaba en público, ya fuera visual o acústicamente. Como es de esperar, este rol se recalcaba todavía a lo largo de las áreas limítrofes de una misma glosa y más allá, entre mayas y no mayas. Nuestros datos parecen indicar prácticas de modificación craneales más uniformes y notables a lo largo de los extremos al oeste, al sur y al sureste de las Tierras Bajas mayas. Algunos de los territorios al este maya y más allá del río Usumacinta se establecieron relativamente tarde por los mayas que estaban en contacto directo con otros pueblos de diversas culturas, 
algunos de los cuales eran claramente no mayas. Concluimos, por lo tanto, que la visualización de la cabeza dentro y entre los diferentes grupos de idiomas mayas debe haber tenido un significado visual para sus portadores humanos, contribuyendo a la construcción a largo plazo de la identidad, que fue impulsado, entre otros factores, por el uso del lenguaje hablado.

Transmitido a través de generaciones de madres y familias, en medio de redes económicas cambiantes, alianzas y competición políticas, estas prácticas se movían junto con las fronteras en expansion y luego en retracción, involucrando a pobladores mayas de diferentes formas de hablar y a no mayas. Algunas de estas identidades socioculturales estaban en franca oposición, una oposición con crecientes implicaciones de hostilidad política y militar entre los dominios mayances de las Tierras Bajas mayas, que culminarían con el denominado «colapso» maya: la caída de las poderosas hegemonías mayas al término de un siglo y el abandono de grandes territorios en el Petén Central y Meridional.

En este contexto, los patrones de distribución de formas cefálicas invitan a realizar nuevas preguntas, de índole más genérica, sobre las condiciones culturales subyacentes.

Las dinámicas conservadoras y cohesivas (centrípetas) del habla se muestran en este tránsito más resilientes que las fuerzas divisoras (centrífugas) del vaivén de las redes económicas, las alianzas políticas y dominios militares. Asimismo, la calidad transgeneracional de las prácticas cefálicas, realizadas y transmitidas por mujeres, condicionó con toda probabilidad su permanencia más allá del colapso. Nuestra conjetura se alinea con el marco conceptual propuesto por Patricia McAnany (1995) al distinguir -a modo de dos polos opuestos- la desigualdad institucionalizada (centrífuga) de la cohesion grupal (centrípeda), que se fundaba en la permanencia de familias, ancestros y tierras. Con el presente ensayo podemos reconocer, además, un componente de género: mientras que las redes económicas y políticas, el comercio y el propio registro epigráfico tendían a ser o eran completamente androcéntricos (dominados por hombres), en cambio, las reproducciones grupales más conservadoras y resilientes, tales como el lenguaje hablado y más aún, el tratamiento de la cabeza infantil y su modificación morfológica parecen haber estado a cargo de las mujeres. La permanencia exitosa en los reductos culturales tras el ocaso del Clásico maya, concede sin duda una ventana única sobre el rol del género en la reproducción a largo plazo y la retención activa de la tradición e identidad grupal.

Agradecimientos. Este trabajo evolucionó de una presentación conjunta en el Annual Meeting de la Society for American Archeology en 2014 y de un capítulo para la obra Social Skins of the Head, que vió la luz en 2018. Las lenguas vernáculas mayas y sus portes cefálicos fue también el tema central de una presentación mía durante el homenaje a Alfonso Lacadena en el Tercer Encuentro Internacional de Gramatología de la UNAM, celebrado en su honor en octubre del 2017, pocos meses antes de su fallecimiento, pérdida irreparable y a destiempo. Si antes teníamos el gran privilegio de la discusión académica abierta y fluida con Alfonso Lacadena, ahora nos compete celebrar y difundir su transcendental legado. Para este trabajo en particular, han sido fundamentales una serie de colaboraciones de proyectos, comunicaciones personales y estudios en centros de investigación, cuyo apoyo y acceso se agradece aquí, y así reconocemos con gratitud las facilidades recibidas del Museo Peabody (Universidad de Harvard), el Centro de Estudios Mexicanos y Centroamericanos (CEMCA) y de la Dirección de Antropología Física (Instituto Nacional de Antropología e Historia). A Rocío Albarrán, Karla Góngora y Karla Arjona agradecemos el apoyo editorial y artístico. 


\section{Referencias}

Ayala, Maricela. 2002. «Acerca de los sistemas sociales, políticos y religiosos de Toniná», en La organización social entre los mayas prehispánicos, coloniales y modernos. Memoria de la Tercera Mesa Redonda de Palenque, Vera Tiesler, Rafael Cobos, y Merle Greene Robertson, eds., pp. 147-162. México: Instituto Nacional de Antropología e Historia, Universidad Autónoma de Yucatán.

Barrera Vásquez, Alfredo, ed. 1980. Diccionario Cordemex. Maya-Español Español-Maya. Mérida: Ediciones Cordemex.

Barrett, Jason y Andrew Scherer. 2005. «Stones, Bones, and Crowded Plazas: Evidence for Terminal Classic Maya Warfare at Colha, Belize». Ancient Mesoamerica 16 (1): 101 118. https://doi.org/10.1017/S0956536105050091.

Barth, Fredrik. 1969. «Ethnic Groups and Boundaries. Introduction», en Ethnic Groups and Boundaries: The Social Organization of Culture Difference, Fredrik Barth, ed., pp. 9-37. Boston: Little, Brown and Co.

Beliaev, Dmitri y Albert Davletshin. 2014. «It Was Then That That Which Had Been Clay Turned into a Man: Reconstructing Maya Anthropogonic Myths». Axis Mundi. Slovak Journal of the Study of Religion 9 (1): 2-12.

Cervera, María Dolores. 2007. «El hetsmek’ como experiencia simbólica de la construcción de los niños mayas yucatecos como personas». Revista Pueblos y Fronteras Digital 4: 1-31. https://doi.org/10.22201/cimsur.18704115e.2007.4.224.

Cetina, Janevi, Evelia Magaña, Belem Ceballos y Amalia Herrera. 2018. «Los portes cefálicos en la iconografía del este mesoamericano. Un recorrido a través de la escultura y la pintura mural», en Modificaciones cefálicas culturales en Mesoamérica. Una perspectiva continental, Vera Tiesler y Carlos Serrano eds., pp. 429-456. México: Instituto de Investigaciones Antropológicas, Universidad Nacional Autónoma de México y Universidad Autónoma de Yucatán.

Chinchilla Mazariegos, Oswaldo, Vera Tiesler, Oswaldo Gómez, y T. Douglas Price. 2015. «Myth, Ritual and Human Sacrifice in Early Classic Mesoamerica: Interpreting a Cremated Double Burial from Tikal, Guatemala». Cambridge Archaeological Journal 25 (1): 187-210. https://doi.org/10.1017/S0959774314000638.

Coe, Michael. 1992. Breaking the Maya Code. Nueva York: Thames and Hudson.

Coe, William R. 1959. Piedras Negras Archaeology: Artifacts, Caches and Burials. Museum Monograph 4. Filadelfia: University Museum, University of Pennsylvania.

Dembo, Adolfo y José Imbelloni. 1938. Deformaciones intencionales del cuerpo humano de carácter étnico. Buenos Aires: Biblioteca Humanior.

Díaz-Andreu, Margarita, Sam Lucy, Staša Babiác y David N. Edwards. 2005. The Archaeology of Identity: Approaches to Gender, Age, Status, Ethnicity, and Religion. Nueva York: Routledge.

Duncan, William. 2009. «Cranial Modification among the Maya: ¿Absence of Evidence or Evidence of Absence?», en Bioarchaeology and Identity in the Americas, Kelly Knudson y Christopher M. Stojanowski, eds., pp. 177-193. Gainesville: University Press of Florida. https://doi.org/10.7183/1045-6635.21.3.290.

Duncan, William y Charles Andrew Hofling. 2011. «Why the Head? Cranial Modification as Protection and Ensoulment among the Maya». Ancient Mesoamerica 22 (1): 199-210. https://doi.org/10.1017/S0956536111000162.

Forné, Mélanie, Arthur Demarest, Horacio Martínez, Paola Torres, Silvia Alvarado y Claudia Arriaza. 2009. «Intercambio y afiliación cultural en Cancuén: la complejidad cultural en las vísperas del Colapso», en XXII Simposio de Investigaciones Arqueológicas en Gua- 
temala 2008, Juan Pedro Laporte, Bárbara Arroyo y Héctor Mejía, eds., pp. 1017-1036. Guatemala: Museo Nacional de Arqueología y Etnología, Asociación Tikal.

García Barrios, Ana y Vera Tiesler. 2011. «El aspecto físico de los dioses mayas». Arqueología Mexicana 112: 59-63.

Gervais, Veronique. 1989. Déformations artificielles de crânes préhispaniques au Guatemala et au Mexique. Tesis Doctoral. Caen: Universidad de Caen.

Gómez, Oswaldo. 1999. «Excavaciones en el interior del Templo V, Tikal», en XII Simposio de Investigaciones Arqueológicas en Guatemala, 1998, Juan Pedro Laporte y Héctor L. Escobedo, eds., pp. 174-182. Guatemala: Museo Nacional de Arqueología y Etnología, Asociación Tikal.

Gronemeyer, Sven. 2014a. The Orthographic Conventions of Maya Hieroglyphic Writing. Tesis Doctoral. Bundoora: Universidad de La Trobe.

- 2014b. «E pluribus unum: Embracing Vernacular Influences in a Classic Mayan Scribal Tradition», en A Celebration of the Life and Work of Pierre Robert Colas, Christophe Helmke y Frauke Sachse, eds., pp. 147-62. Acta Mesoamericana 27. Markt Schwaben: Verlag Anton Saurwein.

Haviland, William y Hattula Moholy-Nagy. 1992. «Distinguishing the High from the Mighty from the Hoi Polloi at Tikal, Guatemala», en Mesoamerican Elites: An Archaeological Assessment, Arlen F. Chase y Diane Z. Chase, eds., pp. 50-60. Norman: University of Oklahoma Press.

Houston, Stephen, John Robertson y David Stuart. 2000. «The Language of Classic Maya Inscriptions». Current Anthropology 41 (3): 321-356. https://doi.org/10.1086/300142.

Houston, Stephen, David Stuart y Karl A. Taube. 2006. The Memory of Bones: Body, Being, and Experience among the Classic Maya. Austin: University of Texas Press.

Hruby, Zachary y Mark Child. 2004. «Chontal Linguistic Influence in Ancient Maya Writing: Intransitive Positional Verbal Affixation», en The Linguistics of Maya Writing, Søren Wichmann, ed., pp. 13-26. Salt Lake City: University of Utah Press.

Jones, Siân. 1997. The Archaeology of Ethnicity: Constructing Identities in the Past and Present. Nueva York: Routledge.

Justeson, John S., William M. Norman, Lyle Campbell y Terrence Kaufman. 1985. The Foreign Impact on Lowland Mayan Language and Script. Middle American Research Institute 53. Nueva Orleans: Tulane University.

Kettunen, Harri. 2005. Nasal Motifs in Maya Iconography. Tesis Doctoral. Helsinki: Universidad de Helsinki.

Lacadena, Alfonso. 2000. «Nominal Syntax and the Linguistic Affiliation of Classic Maya Texts», en The Sacred and the Profane: Architecture and Identity in the Maya Lowlands, Pierre R. Colas, Kai Delvendahl, Markus Kuhnert y Annette Schubart, eds., pp. 111128. Acta Mesoamericana 10. Markt Schwaben: Verlag Anton Saurwein.

Lacadena, Alfonso y Søren Wichmann. 2002. «The Distribution of Lowland Maya Languages in the Classic Period», en La organización social entre los mayas prehispánicos, coloniales y modernos. Memoria de la Tercera Mesa Redonda de Palenque, Vol. 2, Vera Tiesler, Rafael Cobos y Merle Greene Robertson, eds., pp. 275-314. México: Instituto Nacional de Antropología e Historia, Universidad Autónoma de Yucatán.

- 2005. "The Dynamics of Language in the Western Lowland Maya Region», en Art for Archaeological Sake: Material Culture and Style across the Disciplines. Proceedings of the Thirty-Third Annual Chacmool Conference, Andrea Waters-Rist, Christine Cluney, Calla McNamee y Larry Steinbrenner, eds., pp. 32-48. Calgary: Chacmool Archaeological Association, University of Calgary. 
Laporte, Juan Pedro. 1999. «Exploración y restauración en el Conjunto de Palacios de Mundo Perdido, Tikal (Estructura 5C-45/47)», en XII Simposio de Investigaciones Arqueológicas en Guatemala 1998, Juan Pedro Laporte y Héctor L. Escobedo, eds., pp. 183-214. Guatemala: Museo Nacional de Arqueología y Etnología, Asociación Tikal.

- 2005. «Exploración y restauración en la Plataforma Este de Mundo Perdido, Tikal (Estructuras 5D-83 a 5D-89)», en XVIII Simposio de Investigaciones Arqueológicas en Guatemala 2004, Juan Pedro Laporte, Bárbara Arroyo y Héctor Mejía, eds., pp.147193. Guatemala: Museo Nacional de Arqueología y Etnología, Asociación Tikal.

López Austin, Alfredo. 2001. «El núcleo duro, la cosmovisión y la tradición mesoamericana», en Cosmovisión, ritual e identidad de los pueblos indígenas de México, Johanna Broda y Félix Báez-Jorge, eds., pp. 47-65. México: Ediciones Conaculta, Fondo de Cultura Económica.

-. 2009. «El dios en el cuerpo». Dimensión Antropológica 46: 1-34.

Martin, Simon y Nikolai Grube. 2008. Chronicle of the Maya Kings and Queens: Deciphering the Dynasties of the Ancient Maya. $2^{\mathrm{a}}$ ed. Nueva York: Thames and Hudson.

McAnany, Patricia. 1995. Living with the Ancestors. Kinship and Kingship in Ancient Maya Society. Austin: University of Texas Press.

Montes, Javier. 2000. La práctica de la deformación cefálica intencional entre los pobladores prehispánicos de Palenque, Chiapas. Tesis de Licenciatura. México: Escuela Nacional de Antropología e Historia.

Mora Marín, David, Nicholas Hopkins y Kathryn Josserand. 2009. «The Linguistic Affiliation of Classic Lowland Mayan Writing and the Historical Sociolinguistic Geography of the Mayan Lowlands», en The Ch'orti'Maya Area: Past and Present, Brent E. Metz, Cameron L. McNeil y Kerry M. Hull, eds., pp. 15-28. Gainesville: University Press of Florida.

Nuñez, Luis Fernando. 2009. «Temporada 2008: informe de actividades; conjunto habitacional norte, Operaciones 111, 112, 113», en Segundo informe parcial Proyecto Arqueológico Chinikihá temporada 2008, Rodrigo Liendo, ed., pp. 159-221. México: Universidad Nacional Autónoma de México. Informe remitido al Instituto Nacional de Antropología e Historia. http://www.mesoweb.com/resources/informes/Chinikiha2008.html.

—. 2011. «Actividades realizadas en el conjunto F», en Proyecto Arqueológico Chinikihá, tercer informe parcial, temporada 2010, Rodrigo Liendo, ed., pp. 89-136. México: Universidad Nacional Autónoma de México. Informe remitido al Instituto Nacional de Antropología e Historia. http://www.mesoweb.com/resources/informes/Chinikiha2010.html.

Pallán, Carlos. 2009. Secuencia dinástica, glifos-emblema y topónimos en las inscripciones jeroglificas de Edzná, Campeche (600-900 d.C.): implicaciones históricas. Tesis de Maestría. México: Universidad Nacional Autónoma de México.

Pérez, Kadwin. 2018. «Los cuidados infantiles y los instrumentos usados en la modificación cefálica mesoamericana. Nuevas perspectivas para el estudio de género y la crianza a partir del registro de figurillas», en Modificaciones cefálicas culturales en Mesoamérica. Una perspectiva continental, Vera Tiesler y Carlos Serrano, eds., pp. 283-302. México: Universidad Nacional Autónoma de México, Universidad Autónoma de Yucatán.

Quintanilla, Claudia. 2013. Estudio y análisis de los enterramientos humanos del sitio arqueológico Cancún. Tesis de Licenciatura. Guatemala: Universidad de San Carlos de Guatemala.

Ringle, William, Tomás Gallareta y George J. Bey. 1998. «The Return of Quetzalcoatl: Evidence of the Spread of a World Religion during the Epiclassic Period». Ancient Mesoamerica 9 (2): 183-232. 
Romano Pacheco, Arturo. 1979. «El material osteológico humano de Toniná, Chiapas. Estudio morfológico, descriptivo y comparativo», en Toniná, une cité maya du Chiapas (Mexique). Pierre Becquelin y Claude Baudez, eds., pp. 179-192. Études Mesoaméricaines VI (I). México: Centre d'Études Mexicaines et Centraméricaines.

Romano Pacheco, Arturo y María Teresa Jaén Esquivel. 1990. «El material óseo humano procedente de diversos sitios arqueológicos del valle de Ocosingo, Estado de Chiapas», en Toniná, une cité maya du Chiapas (Mexique). Pierre Becquelin y Eric Taladoire, eds., pp. 1661-1687. Études Mesoaméricaines VI (IV). México: Centre d'Études Mexicaines et Centraméricaines.

Sánchez, Mirna Beatríz. 2008. Emblema visual de identidad y distinción: reconstrucción de rostros mayas (fisionomía y rasgos bioculturales). Tesis de Maestría, Facultad de Ciencias Antropológicas. Mérida: Universidad Autónoma de Yucatán.

Saul, Frank P. 1972. The Human Skeletal Remains of Altar de Sacrificios: An Osteobiographic Analysis. Papers of the Peabody Museum of Archaeology and Ethnology 63-2. Cambridge: Harvard University.

Scherer, Andrew K. 2006. «Bioarqueología de El Kinel, La Técnica y Zancudero», en Proyecto Regional Arqueológico Sierra del Lacandón: informe preliminar no. 4, Charles Golden, Andrew K. Scherer y Rosaura Vásquez, eds., pp. 83-94. Guatemala: Dirección General del Patrimonio Cultural y Natural de Guatemala.

- 2007. «Bioarqueología de El Kinel, temporada de campo 2007», en Proyecto Regional Arqueológico Sierra del Lacandón, 2007: informe de la quinta temporada de campo, Andrew K. Scherer, Charles Golden y Rosaura Vásquez, eds., pp. 186-201. Guatemala: Dirección General del Patrimonio Cultural y Natural de Guatemala.

- 2008. «Bioarqueología de Tecolote, temporada de campo 2004 y 2008», en Proyecto Regional Arqueológico Sierra del Lacandón, 2008: informe no. 6, Charles Golden, Andrew K. Scherer y Rosaura Vásquez, eds., pp. 160-181. Guatemala: Dirección General del Patrimonio Cultural y Natural de Guatemala.

- 2015. Mortuary Landscapes of the Classic Maya: Rituals of Body and Soul. Austin: University of Texas Press.

—. 2018. «El ser, la identidad y la cabeza entre los mayas del Clásico de los reinos del río Usumacinta», en Modificaciones cefálicas culturales en Mesoamérica. Una perspectiva continental, Vera Tiesler y Carlos Serrano, eds., pp. 531-558. México: Universidad Nacional Autónoma de México, Universidad Autónoma de Yucatán.

Scherer, Andrew, Cassady Yoder y Lori Wright. 1999. «Los esqueletos de Piedras Negras: reporte preliminar no. 3», en Proyecto Arqueológico Piedras Negras: informe preliminar no. 3, tercera temporada, 1999, Héctor L. Escobedo y Stephen D. Houston, eds., pp. 387-399. Guatemala: Instituto de Antropología e Historia.

Sharer, Robert J. y Loa P. Traxler. 2006a. The Ancient Maya. 6th ed. Stanford: Stanford University Press.

- 2006b. «The Foundations of Ethnic Diversity in the Southwestern Maya Area», en Maya Ethnicity: The Construction of Ethnic Identity from Preclassic to Modern Times, Frauke Sachse, ed., pp. 31-43. Acta Mesoamericana 19. Markt Schwaben: Verlag Anton Saurwein.

Scherer, Andrew y Lori Wright. 2001. «Los esqueletos de Piedras Negras: reporte preliminar no. 4», en Proyecto Arqueológico Piedras Negras: informe preliminar no. 4, cuarta temporada, 2000, Héctor L. Escobedo y Stephen D. Houston, eds., pp. 553-557. Guatemala: Instituto de Antropología e Historia.

Sotelo, Laura Elena y Carmen Valverde. 1992. «Los señores de Yaxchilán: un ejemplo de felinización de los gobernantes mayas». Estudios de Cultura Maya 19: 187-214. 
Stewart, T. Dale. 1953. «Skeletal Remains from Zaculeu, Guatemala», en The Ruins of Zaculeu, Guatemala, Vol. 1, Richard B. Woodbury y Aubrey S. Trik, eds., pp. 295-311. Richmond: William Byrd.

Stuart, David. 1987. Ten Phonetic Syllables. Research Reports on Ancient Maya Writing 14. Washington, D.C.: Center for Maya Research.

Suzuki, Shintaro. 2018. «El modelado cefálico en Copán, Honduras. Una perspectiva multiétnica migratoria en el desarrollo de Copán clásico», en Modificaciones cefálicas culturales en Mesoamérica. Una perspectiva continental, Vera Tiesler y Carlos Serrano, eds., pp. 611-631. México: Universidad Nacional Autónoma de México, Universidad Autónoma de Yucatán.

Taube, Karl A. 2003. «Tetitla and the Maya Presence at Teotihuacan», en The Maya and Teotihuacan. Reinterpreting Early Classic Interaction, Geoffrey Braswell, ed., pp. 273-314. Austin: University of Texas Press.

Tiesler, Vera. 1998. La costumbre de la deformación cefálica entre los antiguos mayas: aspectos morfológicos y culturales. México: Instituto Nacional de Antropología e Historia. . 1999. Rasgos bioculturales entre los antiguos mayas: aspectos arqueológicos y sociales. Tesis Doctoral, Facultad de Filosofía y Letras. México: Universidad Nacional Autónoma de México.

. 2010. "'Olmec' Head Shapes among the Preclassic Period Maya and Cultural Meanings»». Latin American Antiquity 21 (3): 290-311. https://doi.org/10.7183/10456635.21.3.290.

. 2011. «Becoming Maya: Infancy and Upbringing through the Lens of Pre-Hispanic Head Shaping». Childhood in the Past 4 (1): 117-132. https://doi.org/10.1179/ cip.2011.4.1.117.

- 2012. Transformarse en maya: el modelado cefálico entre los mayas prehispánicos y coloniales. Mérida: Universidad Nacional Autónoma de México, Universidad Autónoma de Yucatán.

- 2014. The Bioarchaeology of Artificial Cranial Modifications: New Approaches to Head Shaping and Its Meanings in Pre-Columbian Mesoamerica and Beyond. Nueva York: Springer.

- 2015a. «Shifts in Artificial Head Forms, Population Movements, and Ethnicity among the Postclassic Maya», en Archaeology and Bioarchaeology of Population Movement among the Prehispanic Maya, Andrea Cucina, ed., pp. 143-154. Nueva York: Springer.

- 2015b. Reporte de los restos humanos explorados por el Proyecto Arqueológico La Blanca, Petén, Guatemala. Informe no publicado. Valencia: Universidad de Valencia.

Tiesler, Vera y Andrea Cucina. 2010. «La deformación craneana como emblema de identidad, etnicidad y reproducción cultural entre los mayas del Clásico», en Identidades y cultura material en la región maya, Héctor Hernández Álvarez y Marcos Noé Pool Cab, eds., pp. 111-134. Mérida: Universidad Autónoma de Yucatán.

- 2012. «Where Are the Warriors? Cranial Trauma Patterns and Conflict among the Ancient Maya», en The Bioarchaeology of Violence, Debra L. Martin, Ryan P. Harrod y Ventura R. Pérez, eds., pp. 160-179. Gainesville: University Press of Florida.

Tiesler, Vera y Alfonso Lacadena. 2018. «Head Shapes and Group Identity on the Fringes of the Maya Lowlands», en Social Skins of the Head. Body Beliefs and Ritual in Ancient Mesoamerica and the Andes, Vera Tiesler y $\mathrm{M}^{\mathrm{a}}$ Cecilia Lozada, eds., pp. 37-58. Albuquerque: University of New Mexico Press.

Tovalín, Alejandro, Javier Montes de Paz y José Adolfo Velázquez de León. 1998. «Costumbres funerarias en Bonampak, Chiapas», en XI Simposio de Investigaciones Arqueoló- 
gicas en Guatemala 1997, Juan Pedro Laporte, Héctor L. Escobedo y Sandra Villagrán, eds., pp. 249-260. Guatemala: Museo Nacional de Arqueología y Etnología, Asociación Tikal.

Vargas, Ernesto. 2001. Itzamkanac y Acalan: tiempos de crisis anticipando el futuro. México: Instituto de Investigaciones Antropológicas, Universidad Nacional Autónoma de México.

Webster, David. 2002. The Fall of the Ancient Maya: Solving the Mystery of the Maya Collapse. Nueva York: Thames and Hudson.

Wright, Lori E. 1997. «Los restos óseos humanos de Piedras Negras: un reporte preliminar», en Proyecto Arqueológico Piedras Negras: informe preliminar no. 1, primera temporada, 1997, Héctor L. Escobedo y Stephen D. Houston, eds., pp. 213-218. Guatemala: Instituto de Antropología e Historia.

Wright, Lori E. y Amy M. Witte. 1998. «Observaciones sobre la osteología humana de Piedras Negras: reporte preliminar no. 2», en Proyecto Arqueológico Piedras Negras: informe preliminar no. 2, segunda temporada, 1998, Héctor L. Escobedo y Stephen D. Houston, eds., pp. 393-397. Guatemala: Instituto de Antropología e Historia.

Wyllie, Cherra E. 2002. Signs, Symbols, and Hieroglyphs of Ancient Veracruz: Classic to Postclassic Transition. Tesis Doctoral. New Haven: Yale University.

Zabala, Pilar. 2014. «Source Compilation on Head-Shaping Practices in Prehispanic America», en The Bioarchaeology of Artificial Cranial Modifications: New Approaches to Head Shaping and Its Meanings in Pre-Columbian and Colonial Mesoamerica, Vera Tiesler, ed., pp. 99-129. Nueva York: Springer. 